\title{
Validation of a Vibrating-Wire Viscometer: Measurements in the Range of 0.5 to $135 \mathrm{mPa} \cdot \mathrm{s}$
}

\author{
Fernando J. P. Caetano, ${ }^{\dagger, \ddagger}$ João M. N. A. Fareleira, ${ }^{*, \dagger}$ Carla M. B. P. Oliveira, ${ }^{\dagger, \ddagger}$ and \\ William A. Wakeham ${ }^{\S}$
}

Centro de Química Estrutural, Instituto Superior Técnico, Universidade Técnica de Lisboa, Av. Rovisco Pais, 1049-001 Lisboa, Portugal, Universidade Aberta, R. da Escola Politécnica, 147, 1269-001 Lisboa, Portugal, and University of Southampton, Highfield, Southampton SO17 1BJ, United Kingdom

\begin{abstract}
The present article describes a novel vibrating-wire viscometer that has been shown to be able to measure viscosities up to $135 \mathrm{mPa} \cdot \mathrm{s}$, after calibration against water at $20{ }^{\circ} \mathrm{C}$, to provide direct traceability to the primary reference for viscosity. For the purpose of validating the instrument, measurements of the viscosity of some selected fluids, including standard reference liquids with viscosity on the order of $100 \mathrm{mPa} \cdot \mathrm{s}$ at $20{ }^{\circ} \mathrm{C}$ and 2,2,4-trimethylpentane with a viscosity of about $0.5 \mathrm{mPa} \cdot \mathrm{s}$ at the same temperature, were performed. The results obtained show that the instrument is capable of performing viscosity measurements with an estimated overall uncertainty better than $\pm 0.8 \%$ over the range of $(0.5$ to 135$) \mathrm{mPa} \cdot \mathrm{s}$.
\end{abstract}

\section{Introduction}

There is strong industrial demand for a standard for viscosity that is closer to the value for the fluids most encountered in industrial practice than is the accepted value for the viscosity of water at $20{ }^{\circ} \mathrm{C} .1,2$ As a first step, the Bureau International des Poids et Mesures (BIPM) together with the former Subcommittee on Transport Properties ${ }^{19}$ of the Physical Chemistry Division of IUPAC has initiated a search for a standard reference fluid with a viscosity in the range of (100 to 200$) \mathrm{mPa} \cdot \mathrm{s}^{3}$ One fluid identified for this purpose is diisodecylphthalate (DIDP), which was the subject of an earlier preliminary study. ${ }^{4}$ If such a fluid can be identified, whether DIDP or another, then it would greatly enhance the accuracy, ease, and cost of industrial viscosity measurements. This is because current methodologies require the use of the viscosity of water at $20^{\circ} \mathrm{C}$ as the ultimate standard and the use of up to six capillary viscometers with overlapping ranges to permit the final instrument to reach viscosities around 200 $\mathrm{mPa} \cdot \mathrm{s}$ in a calibrated manner. Evidently, this sequential process leads to an accumulation of errors and expense.

Vibrating-wire instruments are free of several of the limitations present in most capillary devices, such as surface tension effects. In a previous publication, ${ }^{4}$ we presented our first measurements of the viscosity of DIDP, using a novel vibrating-wire instrument after calibration against toluene, at $25^{\circ} \mathrm{C}$ and under atmospheric pressure. The limited accuracy of these first results for the viscosity of DIDP is thought to be attributable to the choice of the reference fluid used to carry out the calibration of the instrument. Therefore, in the new measurements, it was decided to calibrate the effective wire radius with water at $20^{\circ} \mathrm{C}$, which provides direct traceability to the primary reference value for viscosity. Furthermore, significant modifications of the mechanical structure of the measurement cell and the electronic instrumentation are expected to contribute to the improvement of the viscosity results,

\footnotetext{
* To whom correspondence should be addressed. E-mail: j.fareleira@ ist.utl.pt.

Universidade Técnica de Lisboa.

¥ Universidade Aberta.

$\S$ University of Southampton.
}

compared to the results from the previous version of the equipment.

\section{Theoretical Model}

The theoretical description of the hydrodynamic effects on the oscillatory motion of a vibrating-wire sensor operated in forced mode has been published elsewhere; ${ }^{5-7}$ therefore, only a brief description of the working equations is given here.

The resonance frequency, $\omega_{\mathrm{r}}$, and the half-width of the resonance curve for the velocity of the wire, which is what is observed in practice, $\Delta \omega=\omega_{+}-\omega_{-}$, are related to the cell parameters through eqs 1 and 2 :

$$
\begin{gathered}
\frac{\mathrm{d}}{\mathrm{d} \tilde{\omega}}\left\{\tilde{\omega}^{2}\left(\beta^{\prime}+2 \Delta_{0}\right)^{2}+\left[\tilde{\omega}(1+\beta)-\frac{\tilde{\omega}_{0}^{2}}{\tilde{\omega}}\right]^{2}\right\}=0 \\
\frac{\tilde{\omega}_{ \pm}\left(\beta^{\prime}+2 \Delta_{0}\right)}{\tilde{\omega}_{ \pm}^{2}\left(\beta^{\prime}+2 \Delta_{0}\right)^{2}+\left[\tilde{\omega}_{ \pm}(1+\beta)-\frac{\tilde{\omega}_{0}^{2}}{\tilde{\omega}_{ \pm}^{2}}\right]^{2}}= \\
\frac{1}{2} \frac{\tilde{\omega}_{\mathrm{r}}^{2}\left(\beta^{\prime}+2 \Delta_{0}\right)}{\tilde{\omega}_{\mathrm{r}}^{2}\left(\beta^{\prime}+2 \Delta_{0}\right)^{2}+\left[\tilde{\omega}_{\mathrm{r}}(1+\beta)-\frac{\tilde{\omega}_{0}^{2}}{\tilde{\omega}_{\mathrm{r}}}\right]^{2}}
\end{gathered}
$$

In the above equations, the tilde above a symbol indicates a corresponding dimensionless quantity; ${ }^{5,7} \omega_{0}$ represents the natural frequency (in the complete absence of damping), and $\Delta_{0}$ is the wire internal damping, assuming that the axial tension on the wire is the same as for the oscillation in the fluid. The oscillation characteristics of the vibrating-wire sensor are dependent on the density, $\rho$, and viscosity, $\eta$, of the surrounding fluid through the functions $\beta$ and $\beta^{\prime}$, defined as ${ }^{5,7}$

$$
\beta=\left(\frac{\rho}{\rho_{\mathrm{s}}}\right) k \quad \text { and } \quad \beta^{\prime}=\left(\frac{\rho}{\rho_{\mathrm{s}}}\right) k^{\prime}
$$

with

$$
k=-1+2 \mathscr{T} m(A) \quad \text { and } \quad k^{\prime}=2 \operatorname{Re}(A)
$$


and

$$
A=j\left[1+\frac{2 K_{1}(\sqrt{j \Omega})}{\sqrt{j \Omega} K_{0}(\sqrt{j \Omega})}\right]
$$

The quantities $K_{0}$ and $K_{1}$ are modified complex argument Bessel functions, and $\Omega$ is a dimensionless frequency related to the Reynolds number for the fluid motion around the wire:

$$
\Omega=\frac{\rho \omega R^{2}}{\eta}
$$

The present vibrating-wire sensor has been developed to fulfill the following basic requirements: (i) a single sensor should be able to perform measurements of viscosity in the range of $(0.5 \leq \eta \leq 120) \mathrm{mPa} \cdot \mathrm{s}$ and (ii) the measurement precision should be on the order of $\pm 0.5 \%$ over the whole range of operation. For this purpose, simulation studies were performed to select the physical parameters of the cell, namely, the radius and length of the wire sensor, and to determine other operating conditions that ensure an adequate quality factor for the resonance curves. ${ }^{4}$

A detailed analysis of all of the constraints as well as some exploratory tests performed has shown that a suitable set of conditions for the measurement, with a single sensor, of viscosities up to $120 \mathrm{mPa} \cdot \mathrm{s}$ is a tungsten wire with a nominal radius of about $200 \mu \mathrm{m}$ and a length of about 0.06 $\mathrm{m}$. Concerning the operating conditions, it was assumed that the vertical tension applied to the wire should not exceed $50 \%$ of the wire material's yield strength, leading to a resonance frequency on the order of $1 \mathrm{kHz}$. Regarding the ancillary electronic equipment, it has been estimated that the necessary driving current should be less than 30 $\mathrm{mA}$ for the complete range of viscosity.

An additional matter of concern with respect to the design is the selection of the minimum distance from the oscillating wire at which boundaries to the fluid may be placed without affecting the operation of the instrument. As pointed out before, ${ }^{4}$ the closest distance of a cell component from the wire is, for the current measurement cell, on the order of 50 times the wire radius, $R$. For the evaluation of all of the viscosities reported here, we have employed the theory for a wall placed at an infinite distance from the wire. The resulting contribution to the error in the reported viscosities, incurred because of the practical deviation from this assumption, is greatest for the greatest viscosity. An estimate of that contribution, assuming a cylindrical boundary ${ }^{8}$ placed at a distance from the wire of about 50 times its radius, shows that it would not exceed $-0.18 \%$ for a viscosity as large as $135 \mathrm{mPa} \cdot \mathrm{s}$, which sets an upper limit for the present measurements.

\section{Experimental Setup}

Measurement Cell. The vibrating-wire sensor and the measurement cell assembly have been briefly described previously. ${ }^{4}$ Figures 1 and 2 show schematic drawings, respectively, of the cell and of the vessel and the magnetic circuit assembly. The vibrating wire employed is made of tungsten (supplied by Goodfellow Cambridge Limited, U.K.), with a purity of $99.95 \%$ and a nominal radius of 190 $\mu \mathrm{m}$. The wire is clamped at both ends to two Inconel plates that are kept parallel and rigidly fixed by two 4-mmdiameter tungsten rod spacers. Electrical insulation of the wire is obtained using washers machined from Ertacetal

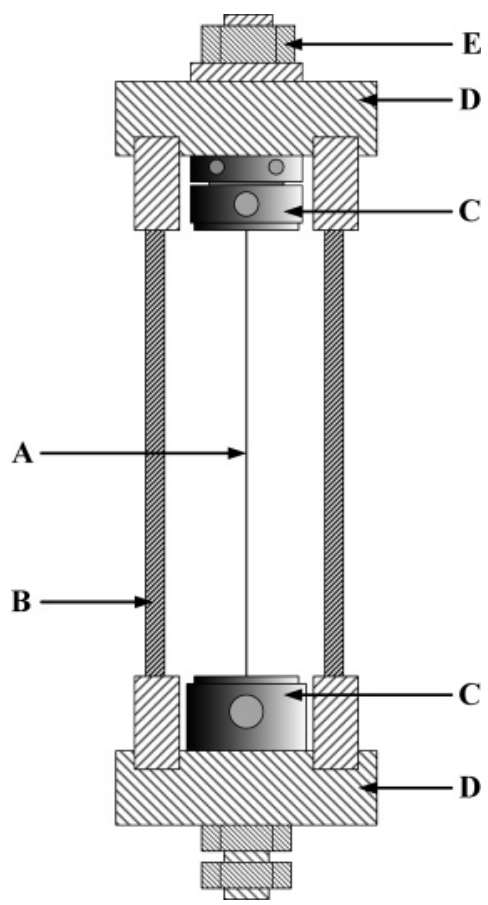

Figure 1. Schematic drawing of the vibrating-wire sensor; A, Tungsten vibrating wire; B, Tungsten rod spacers; C, Inconel clampers for the vibrating wire; D, Inconel plates; E, Fine threaded nut, for tensioning without torsion of the wire.

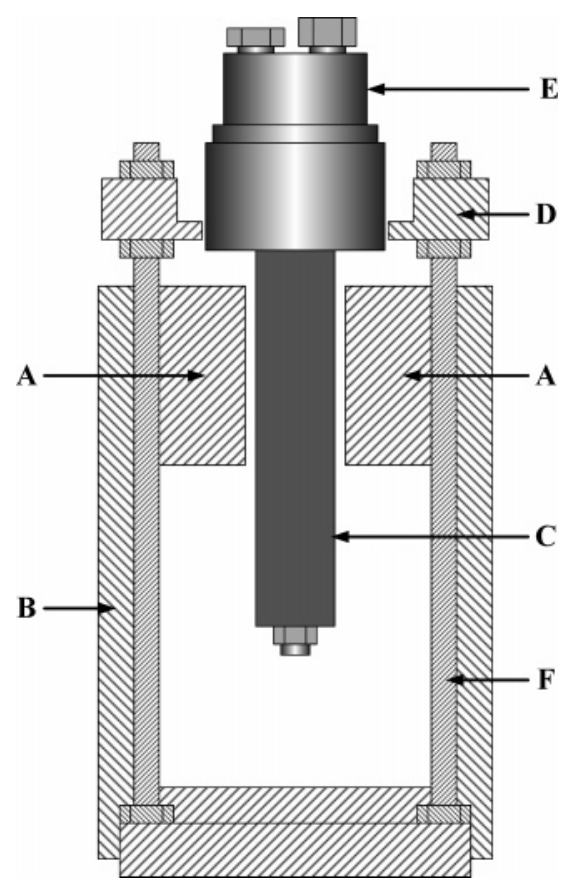

Figure 2. Schematic drawing of the measurement vessel with the vibrating-wire sensor and the magnetic circuit assembly. A, $\mathrm{NdFeB}$ magnets; B, magnetic circuit; C, Stainless steel vessel; D, Stainless steel support ring; E, Vessel cap with feed-through for electrical connections; F, Stainless steel rods to adjust the magnetic circuit position relative to the vibrating wire sensor.

rods (supplied by DSM Engineering Plastic Products, The Netherlands).

The mechanical arrangement was designed to enable the adjustment of the vertical tension on the wire, by means of a finely threaded nut, without torsion of the wire. Similarly, the rotation of the chuck, necessary to minimize any torsional stresses, is made possible without changing the vertical tension. 
The cell is connected to the cap of a custom-made 316 stainless steel vessel (Figure 2). The part of the vessel mounted in the magnetic gap has plane parallel side walls. The distances between the outer surfaces of the parallel walls are $21.5 \mathrm{~mm}$ in the direction of the magnetic field and $38.5 \mathrm{~mm}$ at right angles. The electrical contacts of the vibrating wire are made using four copper leads, two for each end of the wire, that pass through the cap of the vessel by means of a custom-made feed-through.

The magnetic circuit, made of soft iron, is mounted outside and around the vessel. The magnetic field is created by a set of $\mathrm{NdFeB}$ permanent magnets supplied by Sura Magnets (Sweden). The circuit with the magnets is mounted on a device that enables its partial rotation relative to the vessel. The magnetic induction in the center of the gap between the magnetic poles, where the vibrating wire is located, is about $0.6 \mathrm{~T}$.

The assembly is placed in a thermostatic bath, controlled by a Hart Scientific temperature controller (model 2100) using a $100-\Omega$ platinum resistance thermometer as a sensor. A cold fluid is circulated in a coil immersed in the bath by means of a temperature-controlled external circulator. During one typical measurement, the temperature stability is better than $\pm 0.003 \mathrm{~K}$, with a standard deviation from the mean value on the order of $\pm 0.001 \mathrm{~K}$. Temperature was measured using a $100-\Omega$ platinum resistance thermometer, placed in the thermostatic bath, close to the vessel containing the measuring cell. The thermometer was calibrated at the triple point of water and by comparison with a Tinsley $518725-\Omega$ thermometer, previously calibrated at the National Physical Laboratory, U.K. The uncertainty in the temperature readings is estimated to be $\pm 0.01 \mathrm{~K}$.

Instrumentation. For the present measurements, the vibrating wire sensor has been operated in a forced mode such that its frequency response was obtained experimentally. The essential features of the experimental arrangement used for this kind of measurement are abundantly described in the literature. ${ }^{4,7,9}$ They require the passage of an ac current through the wire, sweeping a range of frequencies around its resonance frequency. As the wire is subject to a permanent magnetic field, the current drives transverse oscillations that gives rise to an additional current in the wire whose amplitude is directly related to the wire velocity. Therefore, an analysis of the electrical impedance of the wire, measured along a range of frequencies containing its resonance frequency, enables the determination of the velocity resonance curve for the transverse oscillations of the wire. ${ }^{10}$ It should be noted, however, that the electronic setup and circuitry used for the present measurements differ from those previously described, which were utilized in our first measurements of the viscosity of diisodecylphthalate. ${ }^{4}$ These incorporated a resistance, including a variable resistor, connected in series with the sensor, aimed at enabling a continuous change of the current.

For the present measurements, the circuitry has subsequently been simplified and all of the cable shielding and earth connections have been revised to minimize the electrical noise in the measurements. In particular, the variable resistor has been withdrawn, and as a consequence, the current level of each measurement was set simply by adjusting the driving signal amplitude. As before, a lock-in amplifier (Perkin-Elmer, model 7225) has been used both as a synthesizer of the variable-frequency driving current and as a voltmeter to measure the potential drop across the vibrating-wire sensor. These measurements

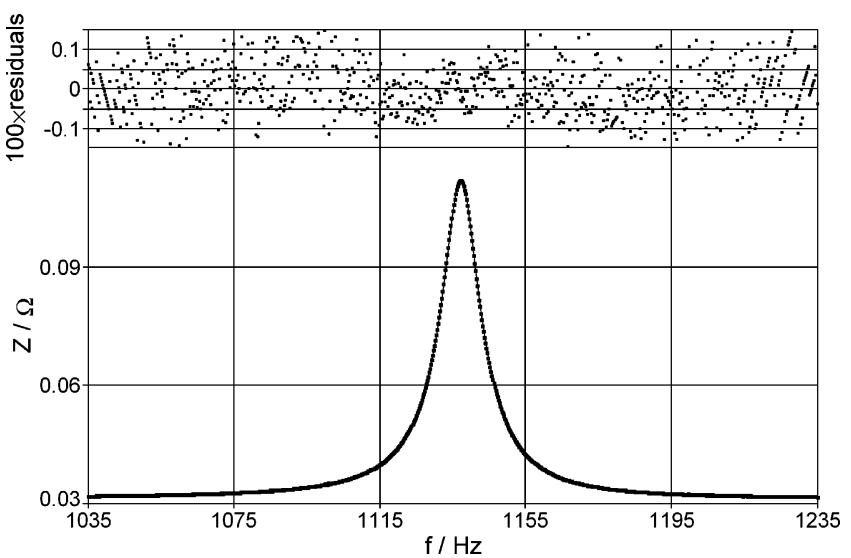

Figure 3. Resonance curve for water at $293.15 \mathrm{~K}$ with a measured viscosity of about $1.002 \mathrm{mPa} \cdot \mathrm{s}$.

were performed using four-lead connections to the differential input plugs and the wave synthesizer output plugs of the lock-in amplifier. A $0.1-\Omega$ standard reference resistor was mounted in series with the sensor to enable the measurement of the electric current passing through the wire, which is necessary to the determination of its impedance. Concerning the driving current variation, it can be seen that for low viscosity values it does not typically exceed $0.2 \%$ of its mean value. This variation is smaller for higher viscosities, and at around $100 \mathrm{mPa} \cdot \mathrm{s}$, the current is constant within $\pm 0.04 \%$.

For the whole range of viscosity tackled in the present measurements, the current supplied to the circuit ranged from about $4 \mathrm{~mA}$ up to $20 \mathrm{~mA}$ to have an adequate response with respect to both the signal-to-noise ratio and the restrictions of the theoretical model. The response of the sensor under vacuum was obtained with a current of about $2 \mathrm{~mA}$. The whole assembly is controlled by a personal computer through an IEEE-488 bus. The data acquired for each resonance curve included the sensor response signal amplitude, phase difference between response and drive signal, and the temperature.

Frequency Response of the Sensor. The response of the wire can be interpreted as a complex impedance, $\bar{Z}$, as described in ref 10 and is given by

$$
\bar{Z}=R_{\mathrm{s}}+i \omega L_{\mathrm{s}}+\frac{1}{G \omega+i \omega C+\frac{1}{i \omega L_{\mathrm{p}}}}
$$

The resonance characteristics of the wire are described by the parallel circuit formed by the capacitor $C$, the inductor $L_{\mathrm{p}}$, and the frequency-dependent conductance $G \omega$. The series resistor, $R_{\mathrm{s}}$, models the circuit resistance, and the inductance, $L_{\mathrm{s}}$, is caused mainly by the measurement vessel feed-through for the electrical leads.

Parameters $C, L_{\mathrm{p}}, L_{\mathrm{s}}, G$, and $R_{\mathrm{s}}$ in eq 7 , which are directly related ${ }^{10}$ to the hydrodynamic model equations, are determined by fitting the experimental resonance curve. The velocity resonance frequency, $\omega_{\mathrm{r}}$, and the half-width of the resonance curve, $\Delta \omega=\omega_{+}-\omega_{-}$, are calculated from the parallel circuit elements; therefore, the viscosity of the fluid surrounding the wire can be calculated by resorting to the hydrodynamic model equations (eqs 1 and 2 ) once the cell parameters and the fluid density are known.

In Figures 3 and 4, two experimental resonance curves are shown, together with the deviations from the fitting equation (eq 7). The fitting procedure and the graphs have been obtained using Table Curve 2D software. Figure 3 shows a resonance curve obtained with water near $20{ }^{\circ} \mathrm{C}$ 


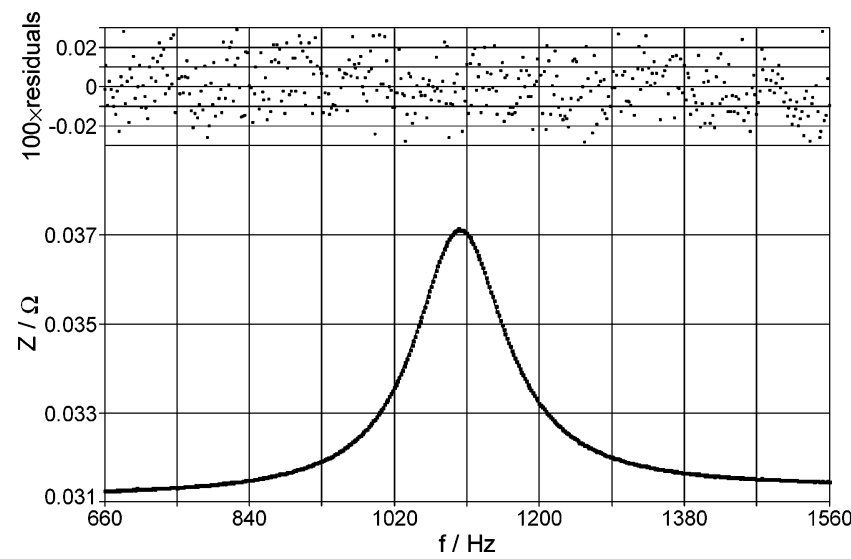

Figure 4. Resonance curve for the Viscosity Standard fluid 100B at $293.15 \mathrm{~K}$ and a measured viscosity of about $125 \mathrm{mPa} \cdot \mathrm{s}$.

and atmospheric pressure, and Figure 4 shows the corresponding curve for a fluid (PTB 100B viscosity standard fluid $^{11}$ ) with a viscosity more than 120 times greater than that of water.

\section{Experimental Details}

Fluids. One of the most serious limitations of the accuracy of the viscosity measurements obtained with the previous vibrating-wire cell was the choice of reference fluid used to carry out the calibration of the instrument. For the present measurements and because of the characteristics of the new cell, the calculation of the effective wire radius is based on water measurements at $20^{\circ} \mathrm{C}$, which provides direct traceability to the primary reference for viscosity.

The water used was previously distilled and deionized by a Millipore system (Milli-Q Plus). The electrical resistivity of the sample was $18.2 \mathrm{M} \Omega \cdot \mathrm{cm}$. Elementary analysis, performed at the Laboratório de Análises of Instituto Superior Técnico (UTL, Portugal) prior to and after the viscosity measurements, showed an iron content of less than $6 \mu \mathrm{g} \cdot \mathrm{dm}^{-3}$ in both instances.

The performance of the equipment at high viscosity was tested with a viscosity standard specimen, PTB 100B (2004-01-26), kindly supplied by Dr. H. Bauer, Physikalisch-Technische Bundesanstalt, Germany, and with the secondary viscosity reference standard material S60 (lot no. 131707, certificate number U0465) supplied by Paragon Scientific Ltd. ${ }^{12}$ These samples were not submitted to any treatment before the measurements.

The low-viscosity performance of the instrument was tested with 2,2,4-trimethylpentane obtained from Merck (UVASOL), with a minimum purity of $99.8 \%$ by GC. The sample was dried using molecular sieves (Riedel-deHaën, $0.4 \mathrm{~nm}$ ), degassed with helium, and introduced into the measuring vessel, under vacuum, without further purification. The water content of the sample prior to the measurements, as determined by Karl Fisher titration, did not exceed $8 \mathrm{mg} / \mathrm{kg}$.

Cell Parameters. The hydrodynamic model equations (eqs 1 and 2) incorporate several parameters that are independent of the fluid, namely, $\Delta_{0}, R$, and the wire density $\rho_{\mathrm{s}}$. Once these and the density of the fluid sample are known, the viscosity of the fluid can be obtained from the parameters of the resonance curve fitting equation (eq 7).

Parameter $\Delta_{0}$ is obtained experimentally from the resonance curve of the sensor under vacuum, ${ }^{7,9}$ and the density of the wire is taken from the literature. ${ }^{13}$
Table 1. Cell Parameters at 293.15 K

$\begin{array}{lll}\text { radius of the wire } & R & 199.68 \mu \mathrm{m} \\ \text { internal damping } & \Delta_{0} & 1.8 \times 10^{-4} \\ \text { wire density } & \rho_{\mathrm{S}} & 19.23 \times 10^{3} \mathrm{~kg} \cdot \mathrm{m}^{-3}\end{array}$

As discussed before, ${ }^{7,9}$ the average radius of the wire is advantageously determined in a single experimental run at a temperature and pressure where the viscosity and density of a reference fluid are well known. Hence, a single experimental run in forced mode with water near $20^{\circ} \mathrm{C}$ and $0.1 \mathrm{MPa}$, which is the primary reference for viscosity, was performed to obtain an effective average radius for the wire sensor. The viscosity and density reference data for water were taken from refs 14 and 15, respectively. The actual cell parameters for the present setup, at $293.15 \mathrm{~K}$, are shown in Table 1.

The value shown for the average wire radius is the mean value of the results provided by nine experimental resonance curves, obtained at temperatures near $20^{\circ} \mathrm{C}$ and 0.1 $\mathrm{MPa}$, the corresponding standard deviation being $0.03 \%$.

Sensitivity analysis indicates that the average wire radius is the most important parameter concerning the overall uncertainty of the viscosity measurements-an uncertainty of $0.1 \%$ in the radius determines by itself an uncertainty in the viscosity of $\pm 0.2 \%$.

The sensitivity of the viscosity results to uncertainties in the other parameters not only is much smaller but also is made practically insignificant as a consequence of the calibration procedure. In particular, a variation of $1 \%$ in the internal damping coefficient, $\Delta_{0}$, typically yields a variation of about $0.08 \%$ in the viscosity results. Similarly, because of the calibration procedure used, an uncertainty in the wire material density affects the final viscosity results mainly through the determination of the wire radius. It is estimated that for viscosities on the order of $100 \mathrm{mPa} \cdot \mathrm{s}$ the overall effect of an uncertainty in the wire density of $\pm 0.2 \%$ leads to an uncertainty of less than $\pm 0.1 \%$ in the viscosity.

\section{Results}

Following the calibration, additional measurements were performed with water over a limited temperature range. Furthermore and in order to assess the quality of the measurements performed with the novel cell, measurements were also carried out on liquids of viscosities on the order of $100 \mathrm{mPa} \cdot \mathrm{s}$ and $1 \mathrm{mPa} \cdot \mathrm{s}$. As mentioned, the highviscosity fluid samples used were secondary reference materials, namely, certified reference fluid S60 (Paragon Scientific Ltd.) and PTB100B viscosity standard specimen (Physikalisch-Technische Bundesanstalt, Braunschweig, Germany). The nominal uncertainties in the certified viscosity of the samples are on the order of $0.27 \%$ and $0.28 \%$, respectively (at a $95 \%$ confidence level), to which the uncertainty of the viscosity of water at $20{ }^{\circ} \mathrm{C}( \pm 0.17 \%)$ should be added. The low-viscosity measurements were performed on a sample of 2,2,4-trimethylpentane, and the results were compared to literature values. All of the measurements were performed under atmospheric pressure. The experiments were performed both in increasing and decreasing cycles of temperature, and no hysteresis was detected within the experimental precision of the measurements.

Table 2 shows the results of the viscosity measurements at several nominal temperatures and under atmospheric pressure. It should be noted that each value is the average of at least six experimental measurements, and the corresponding standard deviation is shown in the third 
Table 2. Experimental Viscosity Measurements as a Function of Temperature and under Atmospheric Pressure of Water, Certified Reference Material S60, and Certified Reference Specimen PTB 100B ${ }^{a}$

\begin{tabular}{lrlrr}
\hline & $T / \mathrm{K}$ & $\eta / \mathrm{mPa} \cdot \mathrm{s}$ & $\sigma / \%$ & $\Delta / \%^{b}$ \\
\hline \multirow{4}{*}{$\mathrm{H}_{2} \mathrm{O}$} & 298.15 & 0.8909 & 0.082 & $0.09^{c}$ \\
& 303.15 & 0.7966 & 0.060 & $-0.07^{c}$ \\
& 288.15 & 1.1397 & 0.023 & $0.10^{c}$ \\
& 298.15 & 0.8912 & 0.062 & $0.13^{c}$ \\
& 303.15 & 0.7969 & 0.097 & $-0.04^{c}$ \\
$\mathrm{~S} 60$ & 293.15 & 135.135 & 0.016 & $0.10^{d}$ \\
& 298.15 & 99.881 & 0.054 & $-0.22^{d}$ \\
& 310.93 & 50.62 & 0.069 & $-0.32^{d}$ \\
\multirow{2}{*}{$100 \mathrm{~B}$} & 293.15 & 125.04 & 0.038 & $0.03^{e}$ \\
& 296.15 & 107.06 & 0.049 & $0.06^{e}$ \\
\multirow{2}{*}{$2,2,4$-trimethylpentane } & 298.15 & 96.901 & 0.029 & $0.11^{e}$ \\
& 293.15 & 124.94 & 0.024 & $-0.05^{e}$ \\
& 298.15 & 0.47601 & 0.013 & $0.02^{f}$ \\
& & & & $0.64^{g}$
\end{tabular}

\begin{abstract}
${ }^{a}$ The viscosity results, $\eta$, are mean values of several measurements, with $\sigma$ being the standard deviation. In the last column, the deviation, $\Delta$, from reference values is shown. ${ }^{b}$ The deviations represent the difference with respect to literature values, as follows: ${ }^{c}$ water ${ }^{15} ;{ }^{d}$ certified reference material S60 ${ }^{12}$; ${ }^{e}$ certified reference specimen PTB $100 \mathrm{~B}^{11}$; f 2,2,4-trimethylpentane ${ }^{16}$; and $g$ 2,2,4-trimethylpentane ${ }^{17}$.
\end{abstract}

column of the Table. The last column presents the deviation of the present average values from the reference data.

The comparison of the present measurements with the reference data, in the defined viscosity range of $(0.5 \leq \eta \leq$ 135) $\mathrm{mPa} \cdot \mathrm{s}$, shows that all of the deviations are within their estimated uncertainty limits. In particular, Table 2 shows that the deviations of the average values obtained for the PTB 100B specimen from its reference data do not exceed $\pm 0.11 \%$ and that the maximum deviation observed with respect to certified reference material S60 is $\pm 0.32 \%$ at $310.93 \mathrm{~K}$. In addition, the measurements carried out with 2,2,4-trimethylpentane show excellent agreement with the data published by Bauer et al. ${ }^{16}$ and by Pádua et al. ${ }^{17}$ at $25{ }^{\circ} \mathrm{C}$, the maximum deviations being $\pm 0.02 \%$ and $0.64 \%$, respectively, well within the corresponding uncertainty limits of their data.

The overall uncertainty of the present measurements is estimated to be better than $\pm 0.8 \%$ over the whole range of viscosity, $(0.5 \leq \eta \leq 135) \mathrm{mPa} \cdot \mathrm{s}$. As a final note, it should be remarked that the performance of the instrument has been further tested with fluids with viscosities higher than the maximum values provided with the certified reference fluids used in the present work. These experiments have indicated that the use of the instrument to measure viscosities up to $200 \mathrm{mPa} \cdot \mathrm{s}$ is possible without a serious loss of precision. This subject will be addressed in detail in a separate publication. ${ }^{18}$

\section{Conclusions}

A new instrument for the measurement of the viscosity of fluids using a vibrating-wire technique in a forced mode of operation that is able to perform measurements in the range $(0.5 \leq \eta \leq 135) \mathrm{mPa} \cdot \mathrm{s}$ has been presented. The instrument requires one calibration operation with a reference fluid, which has been chosen, for the present work, to be water at $20{ }^{\circ} \mathrm{C}$ and under normal pressure, to provide direct traceability to the primary reference point for viscosity. It is hoped that this instrument may contribute to the establishment of a new standard reference fluid for viscosities on the order of $100 \mathrm{mPa} \cdot \mathrm{s}$.

\section{Acknowledgment}

We are grateful to Dr. Harro Bauer, PhysikalischTechnische Bundesanstalt (PTB), Braunschweig, Germany, for the important contribution in discussions and advice concerning this work and for having kindly supplied a highviscosity certified reference liquid sample. We also acknowledge the invaluable help with the electrical measurements from Dr. Pedro Ramos and Dr. Manuel Fonseca da Silva of the Instrumentation and Measurement Group of the Department of Electrical and Computer Engineering of Instituto Superior Técnico, Universidade Técnica de Lisboa, Portugal. Thanks are also due to Dr. J. L. G. Correia da Mata, Academia Militar, Lisbon, Portugal, for his assistance with the initial steps of the work.

\section{Literature Cited}

(1) Swindells, J. F.; Coe, J. R.; Godfrey, T. B. Absolute Viscosity of Water at $20^{\circ} \mathrm{C}$. J. Res. NBS 1952, 48, 1-31.

(2) ISO/TR 3666; Viscosity of Water, 1998.

(3) $19^{\text {th }}$ Meeting of the Sub Committee on Transport Properties of the Commission I.2: Thermodynamics of IUPAC, September 3-4, 1999, Erlangen, Germany.

(4) Caetano, F. J. P.; Fareleira, J. M. N. A.; Oliveira, C. M. B. P.; Wakeham, W. A. Viscosity of Di-isodecylphthalate: A Potential Standard of Moderate Viscosity. Int. J. Thermophys. 2004, 25, 1311-1322.

(5) Retsina, T.; Richardson, S. M.; Wakeham, W. A. The Theory of a Vibrating-Rod Viscometer. Appl. Sci. Res. 1987, 43, 325-346.

(6) Richardson, S. M. Fluid Mechanics; Hemisphere: New York, 1989.

(7) Pádua, A. A. H.; Fareleira, J. M. N. A.; Calado, J. C. G.; Wakeham, W. A. Electromechanical model for vibrating-wire instruments. Rev. Sci. Instrum. 1998, 69, 2392-2399.

(8) Chen, S. S.; Wambsganss, M. W.; Jendrzejczyk, J. A. Added Mass and Damping of A Vibrating Rod in Confined Viscous Fluids. Trans. ASME , Ser. E 1976, 43, 325-329.

(9) Pádua, A. A. H.; Fareleira, J. M. N. A.; Calado, J. C. G.; Wakeham, W. A. Validation of an accurate vibrating-wire densimeter: Density and viscosity of liquids over wide ranges of temperature and pressure. Int. J. Thermophys. 1996, 17, 781-802.

(10) Caetano, F. J. P.; Correia da Mata, J. L. G.; Fareleira, J. M. N. A.; Oliveira, C. M. B. P.; Wakeham W. A. Viscosity Measurements of Liquid Toluene at Low Temperatures Using a Dual VibratingWire Technique. Int. J. Thermophys. 2004, 25, 1-11.

(11) Viscosity Standard Specimens 100B, PTB, Bundesalle 100 D-38116 Braunschweig, 2004-01-26.

(12) Viscosity Reference Standard S60, Lot No. 131707, Certificate of Calibration, Paragon Scientific Limited, Certificate No. U0465, Date of Issue 20-Nov-02.

(13) Oliveira, C. M. B. P. Viscosity of Liquid Hydrocarbons at High Pressure. Ph.D. Thesis, Imperial College of Science, Technology and Medicine, London, 1991.

(14) Bettin, H.; Spieweck, F. Die Dichte des Wassers als Funktion der Temperatur nach Einfuhrung der Internationalen Temperaturskala von 1990. PTB-Mitt 100; 1990; pp 195-196.

(15) Measurement of the Transport Properties of Fluids; Wakeham, W. A., Nagashima, A., Sengers, J. V., Eds.; Experimental Thermodynamics; Blackwell Scientific Publications: Oxford, England, 1991; Vol. 3.

(16) Bauer, H.; Meerlender, G. Precise viscosity measurements of Newtonian liquids with $v<1 \mathrm{~mm}^{2} / \mathrm{s}$ for the selection of suitable standards. Rheol. Acta 1984, 23, 514-521.

(17) Pádua, A. A. H.; Fareleira, J. M. N. A.; Calado, J. C. G.; Wakeham, W. A. Density and Viscosity Measurements of 2,2,4-Trimethylpentane (Isooctane) from $198 \mathrm{~K}$ to $348 \mathrm{~K}$ and up to $100 \mathrm{MPa}$. $J$. Chem. Eng. Data 1996, 41, 1488-1494.

(18) Caetano, F. J. P.; Fareleira, J. M. N. A.; Oliveira, C. M. B. P.; Wakeham, W. A. To be submitted for publication.

(19) Whose efforts have been continued by the informal scientific organization International Association for Transport Properties (IATP).

Received for review July 20, 2004. Accepted October 10, 2004. This work has been supported by Fundação para a Ciência e a Tecnologia (FCT - Portugal) under Programa Operacional "CIÊNCIA, TECNOLOGIA, INOVAÇ̃̃O" (POCTI), with European Union funds (FEDER) and National funds (Project POCTI/ 34951/EQU/2000)

JE049733F 